\title{
Zum Kausalitätsprinzip in der Biologie ${ }^{1}$
}

Kausalität und Determiniertheit sind heuristische Annahmen, die der Sinnhaltigkeit biologischer Experimente dienen. Ihre Setzung als metaphysisches Prinzip im Bereich des Mentalen ist nicht überzeugend begründet.

Mit den neuen neurobiologischen Verfahren ist es möglich geworden, Bewusstseinsvorgänge, die zuvor nur über den Weg der Reportage aus der Perspektive der ersten Person studiert werden konnten, der direkten Untersuchung aus der Perspektive der dritten Person zugänglich zu machen. Die Ergebnisse dieser Forschungsrichtung und mehr noch die Aussichten in die Zukunft, die sie eröffnen, haben zu einem erheblichen Naturalisierungsschub bei der Aufklärung psychologischer und philosophischer Probleme geführt, der eine lebhafte Kontroverse ausgelöst hat.

Ein zentraler Streitpunkt dieser Debatte ist die Frage, ob bewusst erwogene Entscheidungen und ihre Umsetzung in Handlungen frei sein können. Immer wieder wird argumentiert, dass neuronale Prozesse ihr Korrelat in Gehirnprozessen haben und ihrem Wesen nach determiniert ablaufen. Die Freiheit von Willensentscheidung und Handlungsentschluss sei deshalb ein subjektiv konstruiertes Phänomen. Die Möglichkeit, Kausalketten aus dem Bewusstsein heraus in Gang zu setzen, sei ebenso illusionär wie die Vermutung, man könne wirklich auch anders gehandelt haben, hätte sich anders entschieden können. Die zur Debatte stehenden Vorgänge laufen im modular organisierten, massiv parallel informationsverarbeitenden neuronalen Netzwerk des Gehirns ab. Eingeräumt wird, dass diese Prozesse so komplex sind, dass es auf lange Zeit hin und vielleicht für immer unmöglich sein wird, sie genau zu verstehen und vorherzusagen. Das jedoch ist eine Entscheidung, die nicht ,aus dem Lehnstuhl heraus" vorentschieden werden kann, sondern nur als Ergebnis des Forschungsprogramms, das den Phänomenen eine natürliche Beschreibung geben soll. Wenn dies gelungen sein wird, dann wird für die geheimnisvolle kausale Kraft

1 Wiederabdruck des Beitrags von Jens Reich in Naturgeschichte der Freiheit, hrsg. von Jan-Christoph Heilinger, Berlin, New York 2007, S. 29-34. 
des Bewusstseins ebenso wenig Raum sein wie im vergleichbaren Fall für die „vis vitalis“, wenn die physikochemische Beschreibung der Physiologie etwa der Leberoder Nierenfunktion vollständig sein wird. Bewusstsein und freier Wille sind dann alltagstaugliche Zusammenfassungen von emergenten Makroeigenschaften komplexer Vorgänge auf der physikochemischen Mikroebene.

Ebenso wie die meisten anderen Biologen bin ich fasziniert von dem Forschungsprogramm, das kognitive und Bewusstseinsprozesse mit objektiven neurobiologischen Verfahren studiert. Wir werden in den kommenden Jahrzehnten viel Neues erfahren. Es wird auch viel Denkstoff für Anthropologen, Psychologen und Philosophen geben. Konzepte wie die Einheit des individuellen Selbstbewusstseins etwa oder die ursprünglich-synthetische Einheit der Apperzeption (Kant 1781, B 132) wird man auf dem Hintergrund der neuen Tatsache darstellen müssen, dass es im Gehirn keine cartesische Bühne für diese Einheiten gibt.

Verwunderlich erscheint mir allerdings die Leichtigkeit, mit der wir Biologen als Teilnehmer dieser Diskussion dazu neigen, die kausale Determination von einem methodisch-heuristischen Prinzip, das wir erfolgreich bei der Gewinnung und Ordnung von Wissen voraussetzen, in ein metaphysisches Postulat umzusetzen. Ich meine, wir überziehen seinen Geltungsbereich.

Diese letzte Aussage möchte ich in mehrerlei Hinsicht diskutieren und erläutern:

1. Kausaler Determinismus ist methodische Voraussetzung jeder biologischen Erkenntnis (jeder?).

2. Wir leiten unser heuristisches Kausalprinzip aus dem Programm der Reduktion von Biologie auf klassische physikochemische Grundlagen ab, ohne dass wir uns auf die modernen Probleme in diesen Fächern einlassen.

3. Das vom Physikalismus abgezogene Determinismusprinzip ist nur nach a-biologischer Zurichtung des biologischen Objekts verwendbar.

4. Die empirische Unterfütterung des Determinismusprinzips reicht nicht aus, um weitreichende metaphysische Folgerungen plausibel zu machen.

$\mathrm{Zu}$ 1): Es gibt hervorragende biologische Erkenntnis ohne Inanspruchnahme des Determinismus-Postulates. Die klassische Biologie nahm zunächst die Welt, wie sie uns erscheint, und beobachtete. Dieser heute absterbende oder in den Hintergrund rückende Zweig der Biologie bedurfte überhaupt nicht des Postulats, dass jeder Ablauf auch anders verlaufen könnte. Der Anatom ebenso wie der „Naturalist“ beschrieben die Strukturen und Bewegungen, die sie sahen, und konnten ihnen trotz ihrer Regelhaftigkeit durchaus das Erscheinungsbild des nicht notwendig Ablaufenden lassen. Einer der Gipfelpunkte dieser Epoche waren die „Vögel Amerikas“ von John James Audubon - prächtige Zeichnungen und Gemälde von lebenden Tieren in der Illusion der Spontaneität ihres vitalen Verhaltens (Audubon 1994). Nach Ursa- 
chen und Wirkungen wird da nicht gefragt: Stattdessen wird die Kreation wundervoller Phänomene durch die Natur als Kunstwerk beschrieben.

Auch die gesamte Evolutionslehre war zu Anfang eine historische Beschreibung dessen, was vorgefallen war. Sie begann mit den Fossilienfunden vergangener Lebensformen, mit gigantischen Reptilien zum Beispiel, die es zu historischer Zeit nie gegeben hatte, und legte eine sich selbst definierende Zeitskala über die Phänomene (z. B. zur Frage, wie lange es gedauert haben mag, bis eine offensichtlich durch Ablagerung entstandene Kalkschicht entstanden war). Mit Hilfe von Zeitzuordnung und Phänomenologie betrieb man Naturgeschichte, ganz analog wie der Historiker aus Zeitangaben und erinnerten Ereignissen Universalgeschichte ableitet. Selbst Darwins Evolutionstheorie konnte die kausale Beschreibung nur induktiv und extrapolierend einführen, nämlich in Form seines universellen Ausleseprinzips. Sie blieb jedoch offen, da er die verursachenden Agenten für die beobachtete universelle Variation nicht ausfindig machen konnte.

$\mathrm{Zu}$ 2): Determinismus als methodisch notwendiges Postulat trat erst mit dem Siegeszug der experimentellen Biologie in den Vordergrund. Um glaubwürdig zu sein, muss ein biologisches Experiment wiederholbar sein. Hinter dem Postulat der Reproduzierbarkeit steht daher denknotwendig das Postulat der Determiniertheit, nämlich dass sich natürliche Prozesse im Experiment so einrichten lassen, dass sie determiniert ablaufen. Wo das nicht vollständig gelingt, versieht man die Aussage mit statistischen Schwankungsbreiten und anderen Spezifikationen, die das Postulat der Determiniertheit vor Falsifizierung schützen. Eine nicht reproduzierbare Aussage wird ins Reich der grundlosen Spekulation oder bestenfalls der Hypothesen verwiesen.

Die experimentelle Methode ist Teil des Reduktionsprogramms von Biologie auf physikochemische Elementarwelt. Das Determinismuspostulat, das dieses Programm mit sich bringt, setzen wir unkritisch als metaphysisches Prinzip an und sehen über die epistemischen und ontologischen Probleme der modernen Physik hinweg. In der Quantenmechanik zieht sich das Postulat auf die Aussage zurück, dass die Wahrscheinlichkeit von Ereignissen und nicht deren Realisierung streng determiniert sei. Es gibt sogar Wissenschaftler, die das Kausalitätsprinzip der unbelebten Welt ablehnen (wie Bertrand Russell, oder vor ihm Ernst Mach: „In der Natur gibt es keine Ursache und keine Wirkung" [Mach 1883]). Es gibt andere Wissenschaftler, die den gesetzesstrengen Determinismus bestreiten (z. B. Nancy Cartwright „How the Laws of Physics lie“" [Cartwright 1983]). Gegen den strengen kausalen Determinismus ist also bereits in Physik Skepsis angebracht oder zumindest Erklärungsbedarf anzumelden.

$\mathrm{Zu}$ 3): Das gut konzipierte Experiment treibt zuvor dem Leben das Leben aus. Es konstruiert eine geschlossene Teilwelt, die plausibel als von der gesamten Biosphäre 
und des sonstigen Universums hinreichend isoliert angesehen werden darf, und bemüht sich um die Herstellung einer möglichst ausnahmslosen Sukzessionsbeziehung zwischen Auslöser und Effekt. Es gibt kein Experiment, das eine Aussage der Natur, wie sie ist, ermöglicht, sondern eine Aussage, die ihr abgepresst wurde. Oft kann man die Natur zur Antwort zwingen und diese ,verwenden“ - oft jedoch weicht die Natur geschickt einer klaren Antwort aus.

Beim interpretierenden Übergang vom Experiment in die natürliche Welt verflüchtigt sich die Kausalität. Den isolierten Vorgang kann ich noch plausibel als kausale Sukzessionsbeziehung beschreiben, im gesamten System gelingt das nicht mehr. Das ist übrigens bereits in der klassischen Physik der Fall. Jeder der unzähligen Himmelskörper übt die Gravitationskraft auf jeden anderen aus, und jeder ist Ursache der Beschleunigung aller anderen und damit seiner eigenen. So auch in der Biologie: In dem gigantischen Netzwerk von ,wechselwirkenden“ Faktoren, den Genen, den Genprodukten, den niedermolekularen organischen Effektoren, den membranösen Strukturen und dem osmotisch-ionischen wässrigen Milieu, in dem alles Leben sich abspielt, ist für die gradlinige kausale Interpretation kein Platz mehr. Die Schwierigkeit liegt nicht nur darin, dass die vorhandenen Kausalketten so komplex sind, dass Vorhersagen unsicher bis unmöglich werden, analog zum chaotischen Verhalten des Wetters oder zum nicht-linearen Dreikörperproblem in der Physik. Es trifft nicht zu, dass zwar die Vorhersagbarkeit leide, die Kausalität aber intakt sei, sondern schon deren Konstruktion funktioniert überhaupt nicht. Die Imputation von Kausalität erscheint als reine Willkür im Auge des Betrachters. Schon die einfachste Regulationsschleife hat einen Zweig, in dem Vorwärts-,,wirkung“" stattfindet, die durch Rückwärts-,,wirkung“" begrenzt wird. Es ist beliebig, was man hier als Ursache und was als Wirkung klassifiziert, zumal noch dazu alles Geschehen nicht als Sukzession erscheint, sondern im „steady-state“ jedes lebenden Systems gleichzeitig stattfindet. Die mathematische Beschreibung mit der Zeit als laufender Meistervariablen und Evolution nach Setzung von Anfangs- und Randbedingungen ist nur dort adäquat, wo das biologische Experiment als physikalisierter Sonderfall vorliegt. In jeder auf Ganzheitlichkeit zielenden Betrachtung, also der eigentlich adäquaten eines biologischen Systems, müssen wir rekursive Abbildungen von Zustandsfunktionen sowie implizite funktionale Beziehungen zwischen Parametern und Variablenzuständen einführen, wo die Definition von Ursache und Wirkung, also von unabhängigen und abhängigen Größen, reduktionistische Willkür bedeutet. Auf die Spitze formuliert: Es gibt keine metaphysische Kausalität, sondern nur die heuristische und dabei notwendig reduktionistische Anwendung des Kausalprinzips als Beschreibungsform des experimentellen Ansatzes.

$\mathrm{Zu}$ 4): Die Schwierigkeit, von den unzähligen Einzelbefunden auf eine kohärente und schlüssige Gesamtsicht zu kommen, dokumentiert die „Unschärferelation“, dass 
jedes Experiment die Natur verändert oder von ihr hinreichend isoliert ist. Wie immer die Synthese einst gelingen oder misslingen mag, die Übertragung des heuristischen Prinzips der kausalen Bestimmtheit aus der experimentellen Sphäre in den mentalen Bereich ist eine metaphysische Setzung, die nicht zwingend ist.

Ich behaupte also, dass das kausale Gesetzesprinzip in der Biologie sowohl empirisch wie theoretisch unzureichend fundiert ist. Seine Extrapolation in die unüberschaubar komplexe Welt der menschlichen Bewusstseinsphänomene ist nicht zulässig. Dies könnte sich ändern - ich bin kein Mystiker, der ihnen die objektive Erklärbarkeit prinzipiell abspräche. Ignoramus - Ignorabimus: Das erste trifft zu, und das zweite ist Vermutung.

\section{Bibliographie}

Audubon, John J. (1994): Die Vögel Amerikas, Hanau: Werner Dausien.

Cartwright, Nancy (1983): How the Laws of Physics Lie, Oxford, New York: Oxford University Press.

Kant, Immanuel (1781): Kritik der reinen Vernunft, Berlin, New York: Akademie Verlag, 1968.

Mach, Ernst (1883): Die Mechanik in ihrer Entwicklung historisch-kritisch dargestellt, Darmstadt: Wissenschaftliche Buchgesellschaft, 1988. 